\title{
Food intolerance and chronic constipation: manometry and histology study
}

\author{
Giuseppe lacono ${ }^{a}$, Sebastiano Bonventre ${ }^{b}$, Calogero Scalici ${ }^{a}$,Emiliano \\ Maresi $^{\mathrm{c}}$, Lidia Di Prima ${ }^{\mathrm{d}}$, Maurizio Soresi ${ }^{\mathrm{d}}$, Giuseppe Di Gesừ ${ }^{\mathrm{b}}$, Davide Noto ${ }^{\mathrm{d}}$ \\ and Antonio Carroccio ${ }^{d}$
}

Background Chronic constipation in children can be caused by cows' milk intolerance (CMI), but its pathogenesis is unknown.

Aims To evaluate the histology and manometry pattern in patients with food intolerance-related constipation.

Patients and methods Thirty-six consecutive children with chronic constipation were enrolled. All underwent an elimination diet and successive double-blind food challenge. All underwent rectal biopsy and anorectal manometry.

Results A total of 14 patients were found to be suffering from $\mathrm{CMI}$ and three from multiple food intolerance. They had a normal stool frequency on elimination diet, whereas constipation recurred on food challenge. The patients with food intolerance showed a significantly higher frequency of erosions of the mucosa, and the number of intra-epithelial lymphocytes and eosinophils. The rectal mucous gel layer showed that the food-intolerant patients had a significantly lower thickness of mucous than the other subjects studied. Manometry showed a higher anal sphincter resting pressure and a lower critical volume in food intolerance patients than in the others suffering from constipation

\section{Introduction}

Chronic constipation is a very common problem in children [1,2], but despite its high prevalence its cause remains unknown in the majority of cases. Our previous works indicated a clear relationship between cows' milk intolerance (CMI) and chronic constipation in some of the patients $[3,4]$, and it is now accepted that these children can be cured with a cows' milk-free diet. However, the pathogenesis of the CMI-related constipation is unknown. We have recently demonstrated the existence of an inflammatory condition associated with a reduction of the mucous layer on the rectal mucosa, and hypothesized that this second factor could contribute to the pathogenesis of the constipation [5]. However, the aspect of the anorectal motility in these patients has not previously been investigated. In the present study we considered a new series of consecutive patients suffering from chronic constipation and unresponsive to previous standard treatment to evaluate the histology and unrelated to food intolerance. Both histology and manometry abnormalities disappeared on the elimination diet.

Conclusions Food intolerance-related constipation is characterized by proctitis. Increased anal resting pressure and a reduced mucous gel layer can be considered to be contributory factors in the pathogenesis of constipation. Eur J Gastroenterol Hepatol 17:000-000 (c) 2005 Lippincott Williams \& Wilkins.

European Journal of Gastroenterology \& Hepatology 2005, 17:000-000

Keywords: anorectal manometry, chronic constipation, eosinophil cell, food intolerance, rectal histology

aDepartment of Pediatric Gastroenterology, 'Di Cristina' Hospital, Palermo, Italy, Departments of ${ }^{b}$ Surgery, ${ }^{\mathrm{C}}$ Pathology and ${ }^{\mathrm{d}}$ Internal Medicine, University Hospital of Palermo, Palermo, Italy.

Correspondence to Antonio Carroccio, MD, Medicina Interna, Policlinico di Palermo, via del Vespro 141, 90127 Palermo, Italy.

Fax +390916552936; e-mail: acarroccio@hotmail.com

This study was supported by a grant from the MIUR and MiPAF: project 'Alimentazione e celiachia (ALICE)' DD 86 dated 30 January 2002.

Received Accepted

manometry characteristics of the cases of constipation caused by CMI.

\section{Patients and methods}

The study included all the consecutive infants and children with chronic constipation unresponsive to previous treatments examined at the outpatients clinic of the Pediatric Gastroenterology Division of the 'Di Cristina' Hospital, Palermo, Italy, between January and December 2000. Chronic constipation was defined as less than three bowel movement per week with painful elimination of hard stools.

Inclusion criteria were: (i) a history of chronic constipation lasting at least 3 months; (ii) a lack of response to a previous increase in dietary fibre intake or to laxative treatment (milk of magnesia 1-2 $\mathrm{ml}$ per kilogram of body weight); (iii) a regular dietary intake of cow's milk and 
derivatives. Exclusion criteria were: (i) a previous evaluation for chronic constipation; (ii) anatomical/neurological causes of constipation (Hirschsprung's disease one case, psychomotor retardation one case); (iii) constipation as a result of another disease (coeliac disease one case, hypothyroidism one case); (iv) previous anal surgery; (v) the use of medications that can cause constipation; (vi) referral for reasons other than chronic constipation (15 cases).

All patients included were being fed a normal diet, without any restrictions. In accordance with the above criteria 36 patients were recruited (20 females, aged 9 months to 10 years, median 3.6 years), from more than 300 children with chronic constipation, visited during the same period at the outpatients clinic of the hospital and at the private clinic of one of the authors (G.I.). When the patients were first examined a detailed chart was compiled containing the results of the physical examination and case history information.

The infants' parents gave their informed consent to all the diagnostic and therapeutic procedures described in the study. The study protocol was approved by the Ethics Committee of the University Hospital of Palermo.

\section{Study design}

At the first visit in the outpatients clinic, to obtain baseline data (observation period) all medications were stopped and the parents were asked to record for a 2week period the number of bowel movements as well as the appearance of stools and the child's degree of difficulty in passing them, according to a previously validated score $[3,4]$. Furthermore, routine laboratory tests, immunology tests, rectal biopsies and anorectal manometry were performed. At the end of the observation period, the patients underwent a cows' milk-free diet lasting 4 weeks, with the exclusion of cows' milk and its derivatives. During this period, the infants under 15 months old received a formula based on soy (Nutrilonsoya, Nutricia), whereas the children over 15 months received a commercially available soy milk. The patients with persistent constipation on this regimen were placed on a more restricted oligoantigenic diet for a further period of 4 weeks, in the hypothesis of a multiple food intolerance. On the basis of our previous experience [6,7] this diet consisted of rice, lamb, carrots, asses' milk, olive oil, sugar.

After 12 weeks, all the patients who were cured on the cows' milk-free diet or on the more restricted oligoantigenic diet underwent a food challenge. CMI or multiple food intolerance diagnoses were based exclusively on the reappearance of constipation on food challenge and its subsequent disappearance on an elimination diet.
We defined as normalized stool habits a bowel frequency of at least three evacuations per week, with the elimination of soft stools, without painful defecation.

\section{Cows' milk challenge}

The challenges were begun in the hospital according to the procedure previously described [4,6]. As asses' milk had been introduced both into the diet of the patients suffering from multiple food intolerance without causing any clinical reactions, this milk was used as a placebo in performing the double-blind placebo-controlled challenge. The children were randomly assigned to receive cows' milk or placebo. If no clinical reactions were observed within $12 \mathrm{~h}$ after the beginning of the challenge, the patient was discharged and the challenge was continued at home with bottles coded A or B. Neither the parents nor the children were able to distinguish whether the bottles contained asses' or cows' milk. During the 2-week challenge period, the parents recorded any clinical symptoms and the patients were re-examined in the hospital for any adverse reactions. The challenge was stopped when a clinical reaction occurred. The challenges for foods other than cows' milk were performed in open fashion as described elsewhere [5].

\section{Dietary assessment}

To ensure that the children observed a correct elimination diet, the parents were asked to record the amount and the type of food their child had eaten each day. These diaries were analysed at the end of the study to evaluate adherence to the diet and the quantity of milk consumed.

\section{Laboratory test}

At entry to the study routine haematochemical and immunological tests were performed. Methods, reference values and commercial kits employed for serum levels of total IgE, serum levels of $\mathrm{IgE}$ anti-whole milk, casein, lactoalbumin and beta-lactoglobulin (RAST), skin prick tests (PRICK) with food antigens and serum IgG antibetalactoglobulin have been described previously $[4,8]$.

\section{Manometry study}

Anorectal manometry was performed on first observation in all patients. In the patients with food intolerancerelated constipation, the examination was repeated after 12 weeks of the elimination diet, when stool frequency had normalized. Manometry was performed with bowel preparation, when required, using an open perfused catheter; sedation was not necessary. The catheter had a $4.8 \mathrm{~mm}$ external diameter and three radial ways with bearing point. It was perfused with water by a nitrogen infusion pump and rectal distension was produced with a compliant distending rectal balloon attached to the end of the catheter. Data were recorded by an autocalibrating polygraph (Narco Bio System MMS 200; USA) 
connected to a computer with a dedicated software for automatic analysis of the acquired data. In all patients, data recording was begun $10-20 \mathrm{~min}$ after catheter positioning, when the children were calm.

Anal sphincter resting pressure was measured at the end of the manometry procedure and was calculated as the mean of a 3-min period. According to the reference values of our laboratory, derived from anorectal manometry data in healthy age-matched subjects, anal sphincter resting pressure between 45 and $70 \mathrm{mmHg}$ was considered normal.

In children aged over 5 years who collaborated with the medical staff, critical volume was defined as the minimum volume required to produce the sensation of a persistent urge to defecate by filling the rectal balloon with increments of $20 \mathrm{ml}$ per $30 \mathrm{~s}$, reaching a maximum of $300 \mathrm{ml}$. In infants and children aged under 5 years, the critical volume was considered equivalent to that eliciting the anorectal inhibitory reflex. Normal reference values in our laboratory were between 60 and $100 \mathrm{ml}$ (age-matched patients). In all patients, the anorectal inhibitory reflex was tested by distending the rectal balloon, and it was defined as 'normal' when rectal distension caused a relaxation of the anal sphincter pressure of at least $5 \mathrm{mmHg}$.

The medical staff who performed the anorectal manometry were unaware of the clinical and laboratory data of the patients, including the response to the elimination diet, histology data and the immunology study.

\section{Histology study}

Rectal biopsies were performed in all subjects at the end of the 2-week observation period. In the children cured on the elimination diet, a second rectal biopsy was performed after at least 12 weeks of the elimination diet (range 12-14 weeks) when they had normal bowel habits. Endoscopy and histology methods have been described previously [4,5]. Furthermore, the thickness of the surface mucous gel layer was measured according to the method of Matsuo et al. [9] and high iron diamine/alcian blue staining was used to differentiate sulphated mucins from non-sulphated sialomucins [10].

\section{Statistical analysis}

Frequency analysis was performed using Fisher's exact test. Wilcoxon's rank-sum test was used to compare the number of bowel movements per day and the qualitative fecal scores during the baseline period and during the elimination diet period. The same test was used to compare the histology and manometry data recorded in the patients with a final diagnosis of food intolerance, at baseline and after at least 12 weeks of elimination diet, when they were well. The Mann-Whitney U test was used to compare the bowel frequency per day, the qualitative fecal score, the histology and manometry findings in the patients with constipation caused by food intolerance and in those with constipation unrelated to food intolerance. Spearman's $r$ correlation coefficient was used to test the correlation between the histology and manometry data.

\section{Results \\ Clinical data}

After the 2-week observation period, all patients underwent the cows' milk protein-free diet period and during the first 2 weeks of this treatment the number of bowel movements normalized in 14 subjects. The remaining 22 patients were then placed on a more restricted, oligoantigenic diet. On this regimen, bowel movements normalized in another three patients, whereas 19 did not improve. These 19 patients were considered to be suffering from chronic constipation unrelated to food intolerance. The double-blind placebo-controlled cows' milk challenge confirmed that all the 17 patients cured on the elimination diet suffered from cows' milk allergy. In all cases, cows' milk readministration caused the reappearance of constipation, very often associated with abdominal pain, painful defecation and perianal erythema, within 5 days after the commencement of the challenge (median 2 days, range 1-5 days), and these symptoms disappeared on returning to the cows' milkfree diet or to the oligoantigenic diet in the three patients with multiple food intolerance. In these three subjects, other foods were progressively reintroduced into their diet and the following caused the reappearance of constipation: wheat (three cases), egg (two cases), tomato (two cases), fish (two cases), cocoa (one case), soy (one case). For each of these foods, open challenges confirmed intolerance, showing the reappearance of constipation 1-4 days after reintroduction. According to the above results, we diagnosed chronic constipation caused by CMI in 14 children and chronic constipation caused by multiple food intolerance in three patients.

Table 1 shows the number of bowel movements and the qualitative fecal scores in the patients with food intolerance-related chronic constipation before and after the elimination diet and in patients with chronic constipation unrelated to food intolerance. No difference was observed between patients with CMI and patients with multiple food intolerance at baseline either in terms of the number of bowel movements or the qualitative fecal scores.

The subjects with chronic constipation caused by food intolerance showed a higher frequency of a personal history of previous food intolerance $(P=0.01)$ and concomitant signs of food intolerance (bronchospasm four cases, dermatitis two cases) $(P=0.05)$ than the 
Table 1 Number of bowel movements and qualitative fecal scores in patients with food intolerance-related chronic constipation before and after the elimination diet and in patients with chronic constipation unrelated to food intolerance

\begin{tabular}{|c|c|c|c|c|}
\hline & $\begin{array}{l}\text { Patients with food intolerance } \\
\text { before elimination diet }(n=17)\end{array}$ & $\begin{array}{l}\text { Patients with food intolerance } \\
\text { on elimination diet }(n=17)\end{array}$ & $\begin{array}{l}\text { Patients with constipation } \\
\text { unrelated to food intolerance } \\
\qquad(n=19)\end{array}$ & $P$-value \\
\hline \multicolumn{5}{|c|}{$\begin{array}{l}\text { Number of bowel movements/ } \\
\text { week }\end{array}$} \\
\hline Median & $1.5 \mathrm{~A}$ & $5 \mathrm{~A}, \mathrm{~B}$ & $1.5 \mathrm{~B}$ & $A<0.01 B<0.01$ \\
\hline 25 th to 75 th centile & $1-2$ & $3-7$ & $1-2$ & \\
\hline \multicolumn{5}{|l|}{ Qualitative fecal score } \\
\hline 1 & $O A$ & $1 \mathrm{~A}, \mathrm{~B}$ & $\mathrm{OB}$ & \\
\hline
\end{tabular}

In the patients suffering from food intolerance, the elimination diet consisted of a cows' milk protein-free diet in 14 cases and in an oligoantigenic diet in three cases. In patients with constipation unrelated to food intolerance, no difference in the number of bowel movements/week and in the qualitative fecal score was observed between the baseline and the elimination diet periods. Qualitative fecal score: a score of 1 indicates mushy or liquid stools, a score of 2 soft feces and no pain on passing stools, and a score of 3 hard stools and difficulty and pain on passing stools.

Table 2 Clinical and laboratory characteristics at baseline in patients suffering from chronic constipation caused by cows' milk protein intolerance or multiple food intolerance (group 1) and in patients not suffering from food intolerance (group 2)

\begin{tabular}{|c|c|c|c|}
\hline & Group $1(n=17)$ & Group $2(n=19)$ & $P$-value \\
\hline Age (months) & $49.8 \pm 21.2$ & $54.1 \pm 15.2$ & NS \\
\hline Sex (males/females) & $7 / 10$ & $9 / 10$ & NS \\
\hline Breast feeding at birth $(n)$ & $15 / 17$ & $17 / 19$ & NS \\
\hline Duration of illness (months) & $9.4 \pm 3.2$ & $8.9 \pm 3.4$ & NS \\
\hline Family history of food intolerance $(n)$ & $6 / 17$ & $3 / 19$ & NS \\
\hline Personal history of food intolerance $(n)$ & $12 / 17$ & $1 / 19$ & 0.01 \\
\hline $\begin{array}{l}\text { Concomitant symptoms of food intolerance: bronchospasm, } \\
\text { dermatitis, rhinitis }(n)\end{array}$ & $6 / 17$ & $0 / 19$ & 0.05 \\
\hline Soiling or encopresis $(n)$ & $0 / 17$ & $1 / 19$ & NS \\
\hline Abdominal pain $(n)$ & $16 / 17$ & $14 / 19$ & NS \\
\hline Anal fissures or perianal erythema-oedema $(n)$ & $14 / 17$ & $18 / 19$ & NS \\
\hline \multicolumn{4}{|l|}{ Abnormal test results $(n)$} \\
\hline Peripheral eosinophil count & $3 / 17$ & $1 / 19$ & NS \\
\hline Serum IgE level & $3 / 17$ & $1 / 19$ & NS \\
\hline Skin test with food antigens (PRICK) & $3 / 17$ & $0 / 19$ & NS \\
\hline Specific IgE antibodies to food antigens (RAST) & $2 / 17$ & $0 / 19$ & NS \\
\hline Serum IgG anti-betalactoglobulin & $9 / 17$ & $0 / 19$ & 0.05 \\
\hline
\end{tabular}

Values expressed as mean $\pm S D$. Fisher's test was used to compare the frequency in the two groups.

others studied (Table 2). In particular, 12 of the 17 patients with constipation caused by food intolerance suffered from other manifestations of CMI (infantile colics, gastro-oesophageal reflux, chronic diarrhoea with malabsorption, atopic dermatitis) during the first year of life. In all cases, these manifestations disappeared after a period of a cows' milk-free diet and the patients had reassumed foods containing cows' milk proteins.

IgG anti-betalactoglobulin assay was more frequently positive in individuals with constipation caused by food intolerance.

None of the patients had alterations in liver and kidney function or in the indicators of metabolism or inflammation (erythrocyte sedimentation rate, C-reactive protein) during the study. Furthermore, an analysis of the daily calory intake and of the main constituents of the diet did not show any qualitative or quantitative variations during the study period.

\section{Manometry data}

The anal sphincter resting pressure was significantly higher in the patients with constipation caused by food intolerance than in patients with constipation unrelated to food intolerance $(P=0.05)$. At baseline 15 of the 17 patients with food intolerance-related constipation had anal sphincter resting pressures above the higher cutoff value for our laboratory $(70 \mathrm{mmHg})$, but on the elimination diet only one of the 17 patients showed values higher than $70 \mathrm{mmHg}(P<0.001)$. Furthermore, in patients with chronic constipation caused by food intolerance, this parameter was significantly higher at baseline than at the subsequent examination, when the patients were on the elimination diet $(P<0.01)$.

The patients with food intolerance showed at baseline a lower critical volume (minimum volume required to produce the sensation of an urge to defecate) than the patients with constipation unrelated to food intolerance $(P=0.05)$. However, in the patients suffering from food 
Table 3 Anorectal manometry results in patients with food intolerance-related chronic constipation at entry to the study and after 12 weeks of elimination diet, and in patients with chronic constipation not related to food intolerance

\begin{tabular}{|c|c|c|c|c|}
\hline & $\begin{array}{l}\text { Patients with food intolerance } \\
\text { before elimination diet }(n=17)\end{array}$ & $\begin{array}{l}\text { Patients with food intolerance } \\
\text { on elimination diet }(n=17)\end{array}$ & $\begin{array}{l}\text { Patients with constipation } \\
\text { unrelated to food intolerance } \\
\qquad(n=19)\end{array}$ & $P$-value \\
\hline $\begin{array}{l}\text { Anal sphincter resting pressure } \\
\quad(\mathrm{mmHg})\end{array}$ & $88.8+17.6$ A, B & $62.3+7.1 \mathrm{~A}$ & $69.8+14.7 \mathrm{~B}$ & $A<0.01 B<0.05$ \\
\hline Critical volume (ml) & $58.1+13.1 \mathrm{~A}, \mathrm{~B}$ & $73.4+13.2 \mathrm{~A}$ & $69.5+17.3 \mathrm{~B}$ & $A<0.01 B<0.05$ \\
\hline
\end{tabular}

No difference was observed between the 14 patients with cows' milk intolerance and the three patients with multiple food intolerance for any of the manometry parameters considered, either at baseline or on the elimination diet. Normal range values were: for anal sphincter resting pressure $50-70 \mathrm{mmHg}$, for critical volume $60-$ $100 \mathrm{ml}$.

intolerance there was a significant increase in the critical volume on the elimination diet over baseline values $(P=0.01)$. In patients with food intolerance, values of critical volume lower than the minimum cutoff were recorded in eight out of 17 cases at baseline and only in one case on the elimination diet $(P=0.03)$. There was no difference in manometry data between the 14 patients with CMI and the three patients with multiple food intolerance, either at first examination (baseline) or on the elimination diet. Furthermore, we did not find any difference in critical volume values between the older children, who were able to collaborate during the examination, and those of the younger children, either at baseline or at the second examination (Table 3).

\section{Histology data}

At entry to the study, endoscopy showed mild rectal inflammation with mucosal erythema and friability, without ulcerations or erosions in all the 17 patients with food intolerance and in two of the 19 with constipation unrelated to food intolerance $(P<0.001)$. Haematoxylineosin staining showed erosions of the mucosa in 16 out of 17 patients with food intolerance and in only one out of 19 of the subjects who did not suffer from food intolerance $(P<0.001)$. There was no crypts distortion or branching. In the patients with food intolerance, inflammation was characterized by mucosal infiltration of eosinophils, lymphocytes and plasma cells, with the aspect of both follicular and diffuse inflammation (Fig. 1). Patients with food intolerance showed a significantly higher number of intra-epithelial lymphocytes and eosinophils and of eosinophils in the lamina propria than the patients with constipation unrelated to food intolerance (Table 4). On the elimination diet, all the 17 patients with food intolerance-related constipation showed normal rectal endoscopy. At this time, histology did not show mucosa erosions in any of the cases and was absolutely normal in eight of the 17 patients and inflammation was greatly reduced in the others (Fig. 2). Morphometry revealed a significant decrease in the number of intra-epithelial lymphocytes and eosinophils and of eosinophils in the lamina propria over baseline.

The study of the surface mucous gel layer on rectal mucosa, performed at entry to the study, showed that the patients suffering from food intolerance had a marked
Fig. 1

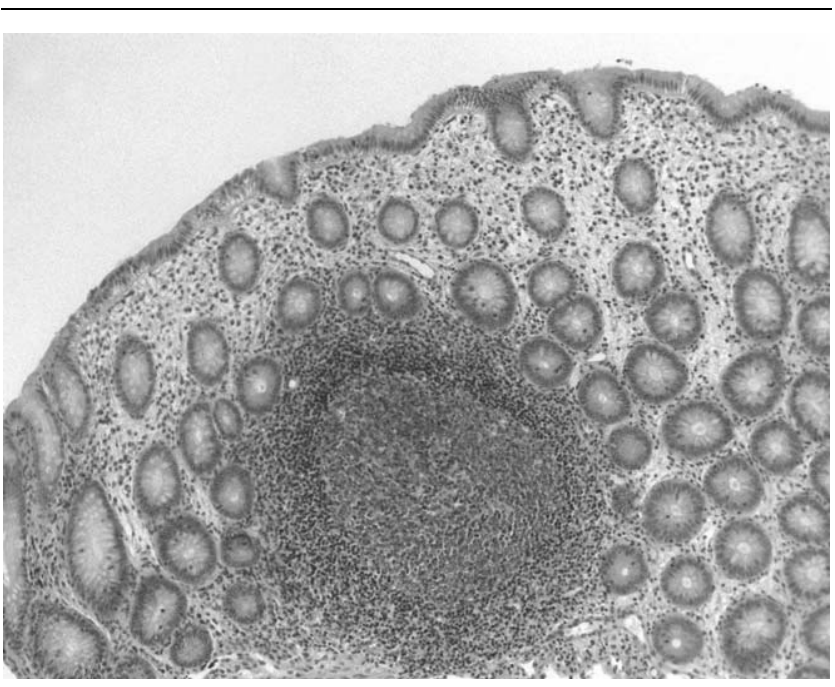

Haematoxylin-eosin staining of the rectal mucosa specimen in a patient with food intolerance-related constipation at diagnosis (before the elimination diet).

reduction in the thickness of the mucous gel layer. There was a reduction or disappearance of the non-sulphated sialomucins in most cases $(14 / 17)$, whereas sulphated mucins were reduced in two out of 17 cases. Only two of the 19 patients with constipation unrelated to food intolerance showed a reduction in the thickness of the mucous gel layer on rectal mucosa. On the elimination diet, the subjects suffering from food intolerance showed a significant increase in the thickness of the mucous gel layer over baseline values.

Finally, we recorded significant correlations between the histology inflammatory parameters and the manometry data (Table 5).

\section{Discussion}

Although our and others' studies have shown the relationship between cows' milk protein intolerance (CMPI) and chronic constipation [3-5,11-13], very little is known about the pathogenesis of chronic constipation caused by CMPI. Consequently, we performed this study 
Table 4 Histology findings in the rectal mucosa of 17 patients with food intolerance-related constipation at entry into the study (group 1 ) and after at least 12 weeks of the elimination diet (group 2) and in 19 patients with constipation unrelated to food intolerance (group 3 )

\begin{tabular}{|c|c|c|c|c|}
\hline & Group $1(n=17)$ & Group $2(n=17)$ & Group $3(n=19)$ & $P$-value \\
\hline Intra-epithelial lymphocytes $(x+S D)$ & $4.8+1.3 \mathrm{~A}, \mathrm{~B}$ & $3.1+0.6 \mathrm{~A}$ & $3.0+0.8 \mathrm{~B}$ & $A=0.02 B=0.005$ \\
\hline Intra-epithelial eosinophils $(x+\mathrm{SD})$ & $4.4+1.5 \mathrm{~A}, \mathrm{~B}$ & $1.2+1.1 \mathrm{~A}$ & $0.9+0.4 \mathrm{~B}$ & $\mathrm{~A}<0.001 \mathrm{~B}<0.001$ \\
\hline Eosinophils in the lamina propria $(x+S D)$ & $8.9+5.6 \mathrm{~A}, \mathrm{~B}$ & $3.8+2.8 \mathrm{~A}$ & $3.4+2.4 \mathrm{~B}$ & $A<0.001 B<0.001$ \\
\hline Thickness of mucous gel layer $(x+\mathrm{SD})$ & $115+55 \mathrm{~A}, \mathrm{~B}$ & $325+190 \mathrm{~A}$ & $340+140 B$ & $A<0.001 B<0.001$ \\
\hline
\end{tabular}

No difference was observed between 14 patients with cows' milk intolerance and three patients with multiple food intolerance for any of the histology parameters considered, either at baseline or on the elimination diet. Intraepithelial lymphocytes and eosinophils were counted per 100 deep crypt cells. The number of eosinophils in the lamina propria was given as a percentage per 1000 lamina propria cells per section. The thickness of the mucous gel layer was expressed in $\mu$ m.

Fig. 2

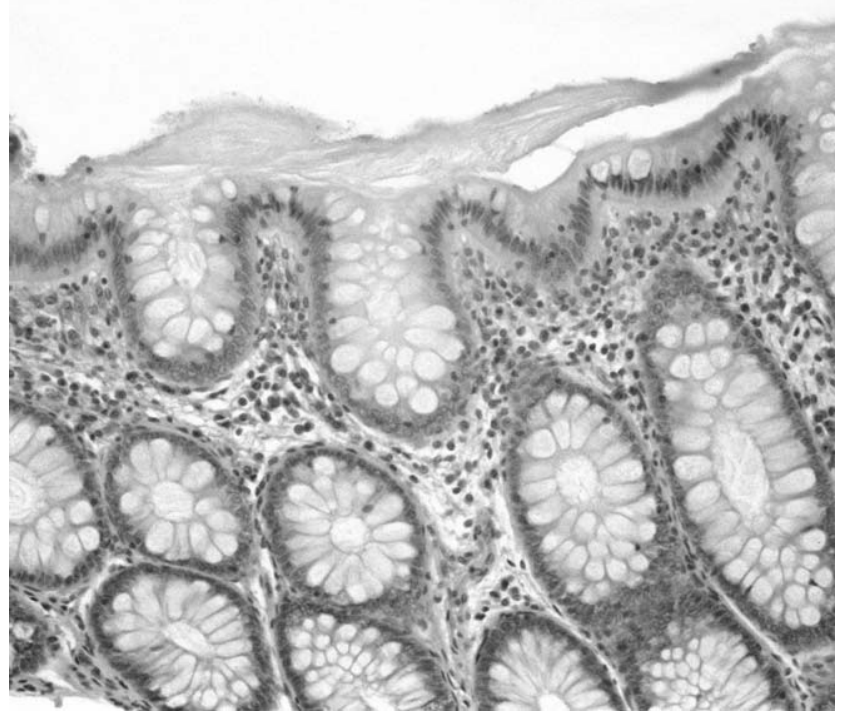

Haematoxylin-eosin staining of the rectal mucosa specimen in a patient with food intolerance-related constipation after the elimination diet.

to investigate the histology aspects and anorectal manometry behaviour in patients with chronic constipation caused by CMPI.

Seventeen of the 36 patients $(48 \%)$ who completed the study showed CMI-related or multiple food intolerancerelated chronic constipation. In these subjects, the symptoms disappeared on the cows' milk-free or oligoantigenic diet and subsequent food challenges demonstrated that these patients were suffering from CMI or multiple food intolerance. In fact, in these cases constipation reappeared when the various foods were reintroduced and disappeared on the elimination diet. According to the European Society Pediatric Gastroenterology Hepatology and Nutrition diagnostic criteria for food intolerance/allergy [14], we can affirm that half of the patients included in the study (17 out of 36 ) were suffering from food intolerance that caused chronic constipation. As studies from other centres showed a frequency of constipation caused by CMI ranging between 28 and $70 \%$ [11-13], it can be concluded that in patients unresponsive to conventional treatments or with a personal history of CMI, constipation is quite frequently a manifestation of food intolerance.

In our series, the immunological assays performed to provide evidence of IgE-mediated hypersensitivity were very often negative, whereas the $\operatorname{IgG}$ anti-betalactoglobulin assay was positive in more than $50 \%$ of cases. This result is in keeping with the reported lack of sensitivity of specific IgE assay in patients with food intolerance and irritable bowel syndrome-like symptoms [15] and with the recent observation that $\operatorname{IgG}$ antibodies to food help to identify dietary intolerances [16]. Furthermore, the observation that the constipation reappeared as a delayed clinical reaction (in mean $48 \mathrm{~h}$ after cows' milk reintroduction) indicates that cell-mediated hypersensitivity seems to be the more probable immune mechanism. This seems to be confirmed by histology: the rectal biopsies of the patients suffering from chronic constipation caused by food intolerance showed inflammation in all subjects, with mucosa erosions in 16 of the 17 patients. These alterations were clearly food dependent as they disappeared completely on the elimination diet. The behaviour of the mucous gel layer of the rectal mucosa is also interesting: our data showed that in food-intolerant patients there was a severe reduction in the thickness of the mucous layer on the rectal mucosa. In patients with food intolerance, mucous reduction was food intolerance dependent, as the thickness of the mucous layer significantly increased on the elimination diet. It is noteworthy that the loss of the mucous barrier function is typical of the inflammatory bowel diseases, and can facilitate the exposure of the mucosa to luminal agents that perpetuate the inflammation $[17,18]$.

The results of the manometry study added convincing data to the hypothesis that food intolerance constipation is related to proctitis. The patients suffering from food intolerance-related constipation had a lower critical volume than patients not suffering from food intolerance when their rectums were distended by insufflation. Once again, reduced rectal compliance is another typical feature of inflammatory bowel diseases. However, the obvious consequence of rectal mucosa inflammation would be chronic diarrhoea caused by sensorial nervous 
Table 5 Correlation between histology and manometry data in all cases included in the study

\begin{tabular}{lccccc}
\hline & Critical volume & $\begin{array}{c}\text { Intra-epithelial } \\
\text { lymphocytes }\end{array}$ & $\begin{array}{c}\text { Inta-epithelial eosinophils } \\
\text { Lamina propria } \\
\text { eosinophils }\end{array}$ & Mucous layer thickness \\
\hline Anal sphincter pressure & $r=0.286 P=0.05$ & $r=0.425 P<0.001$ & $r=0.630 P<0.0001$ & $r=0.625 P<0.0001$ & $r=-0.351 P=0.002$ \\
Critical volume & & $r=0.78 \mathrm{NS}$ & $r=-0.371 P=0.001$ & $r=-0.381 P<0.001$ & $P=0.172 \mathrm{NS}$ \\
Intra-epithelial lymphocytes & & $r=0.633 P<0.0001$ & $r=0.586 P<0.0001$ & $r=-0.513 P<0.0001$ \\
Intra-epithelial eosinophils & & & $r=0.703 P<0.0001$ & $r=-0.639 P<0.0001$ \\
Lamina propria eosinophils & & & & $r=-0.501 P<0.0001$ \\
\hline
\end{tabular}

Spearman's $r$ coefficient of correlation was calculated.

hyperstimulation, and we would ask why does this not happen in cows' milk allergic patients with chronic constipation. The other manometry data we studied could partly explain the cause of the constipation. We found that the anal sphincter resting pressure of the patients with food intolerance was higher than normal, at baseline, in 15 out of 17 patients and the mean value was significantly higher in these patients than in those not suffering from food intolerance. Once again, follow-up showed that on the elimination diet the anal sphincter pressure significantly decreased and normalized in almost all patients with food intolerance. Furthermore, we showed a direct relationship between food intolerance, the intensity of the mucosal inflammation and the anal sphincter pressure. It is possible that the pathogenesis of the constipation in food-intolerant individuals depends on a complex phenomenon such as 'allergic dysmotility'. Our findings have shown eosinophil infiltrate to be the main histology characteristic of proctitis in patients with constipation caused by food intolerance and eosinophil infiltration of the mucosa significantly correlated with manometry data. The eosinophil is the cell type most clearly associated with allergic dysmotility also in other tracts of the gastrointestinal tube $[19,20]$. Furthermore, there has been recent confirmation that eosinophil recruitment could disrupt enteric nerve function in sensitized mice, as electron microscopy showed areas of neural damage adjacent to degranulating eosinophils in the gastric mucosa [21]. Also noteworthy is the correlation between the eosinophils infiltrate and the reduction in the mucous gel layer on the rectal mucosa. We speculate that in patients with chronic constipation the loss of the adherent rectal mucous layer reduces the physiological lubricating role, and could contribute to the pathogenesis of the constipation.

In summary, the main conclusions of our study were: (i) chronic constipation in children can be caused both by CMI and multiple food intolerance; (ii) the constipation of food-intolerant patients is associated with proctitis; (iii) increased anal resting pressure and a reduced mucous gel layer can be considered to be contributory factors in the pathogenesis of the constipation.

\section{Acknowledgement}

The authors are grateful to M. Pescatori, MD, University of Rome, for his precious revision of the manuscript.
They would also like to thank Miss Carole Greenall for her revision of the English.

\section{Conflict of interest}

XXX

Authors' contributions

XXX

\section{References}

1 Loening-Baucke V. Constipation in children. Curr Opin Pediatr 1994; 6:556-561.

2 Issenman RM, Hewson S, Pirhonen D, Taylor W, Tirosh A. Are chronic digestive complaints the result of abnormal dietary patterns? Diet and digestive complaints in children at 22 and 40 months of age. Am J Dis Child 1987; 141:679-682.

3 lacono G, Carroccio A, Cavataio F, Montalto G, Cantarero MD, Notarbartolo A. Chronic constipation as a symptom of cow milk allergy. J Pediatr 1995; 126:34-39.

4 lacono G, Cavataio F, Montalto G, Florena A, Soresi M, Tumminello M, et al. Intolerance of cow's milk and chronic constipation in children. $N$ Engl J Med 1998; 338:1100-1104.

5 Carroccio A, Scalici C, Maresi M, Di Prima L, Cavataio F, Noto D, et al. Chronic constipation and food intolerance: a model of proctitis causing constipation. Scand J Gastroenterol 2005; 40:33-42.

6 Carroccio A, Cavataio F, Montalto G, D'Amico D, Alabrese L, lacono G. Intolerance to hydrolised cow's milk proteins in infants: clinical characteristics and dietary treatment. Clin Exp Allergy 2000; 30:15971603.

7 lacono G, Carroccio A, Cavataio F, Montalto G. Multiple food allergy. J Pediatr Gastroenterol Nutr 1993; 16:472-473.

8 lacono G, Carroccio A, Cavataio F, Montalto G, Lorello D, Kazmierska I, et al. IgG anti-betalactoglobulin (betalactotest): its usefulness in the diagnosis of cow's milk allergy. Ital J Gastroenterol 1995; 27: 355-360.

9 Matsuo K, Ota H, Akamatsu T, Sugiyama A, Katsuyama T. Histochemistry of the surface mucous gel layer of the human colon. Gut 1997; 40:782-789.

10 Spicer SS. Diamine methods for differentiating mucopolysaccharides histochemically. J Histochem Cytochem 1965; 13:211-234.

11 Daher S, Sole D, de Morais MB. Cow's milk and chronic constipation in children. N Engl J Med 1999; 340:891.

12 Shah N, Lindley K, Milla P. Cow's milk and chronic constipation in children. $N$ Engl J Med 1999; 340:891-892.

13 Daher S, Tahan S, Solè D, Naspitz CK, Patricio FRS, Fagundes-Neto U, Morais MB. Cow's milk protein intolerance and chronic constipation in children. Pediatr Allergy Immunol 2001; 12:339-342.

14 The European Society for Pediatric Gastroenterology and Nutrition. Working Group for the Diagnostic Criteria for Food Allergy. Diagnostic criteria for food allergy with predominantly intestinal symptoms. J Pediatr Gastroenterol Nutr 1992; 14:108-112.

15 Zar S, Kumar D, Benson M. Review article: food hypersensitivity and irritable bowel syndrome. Aliment Pharmacol Ther 2001; 15:439-443.

16 Atkinson W, Sheldon TA, Shaath N, Whoewell PJ. Food elimination based on $\lg G$ antibodies in irritable bowel syndrome: a randomised controlled trial. Gut 2004; 53:1459-1464.

17 Smithson JE, Campbell A, Andrews JM, Milton JD, Pigott R, Jewell DP. Altered expression of mucins throughout the colon in ulcerative colitis. Gut 1997; 40:234-240.

18 McCormick DA, Horton LWL, Mee AS. Mucin depletion in inflammatory bowel disease. J Clin Pathol 1990; 43:143-146.

19 Cavataio F, lacono G, Montalto G, Soresi M, Tumminello M, Campagna P, et al. Gastroesophageal reflux associated with cow's milk allergy in infants: 
8 European Journal of Gastroenterology \& Hepatology 2005, Vol 17 No 00

which diagnostic examinations are useful? Am J Gastroenterol 1996; 91:1215-1220.

20 Ravelli AM, Tobanelli P, Volpi S, Ugazio AG. Vomiting and gastric motility in infants with cow's milk allergy. J Pediatr Gastroenterol Nutr 2001; 32:5964.
21 Hogan SP, Mishra A, Brandt EB, Royalty MP, Pope SM, Zimmermann N. A pathological function for eotaxin and eosinophils in eosinophilic gastrointestinal inflammation. Nat Immunol 2001; 2:353-360. 


\section{AUTHOR QUERY FORM}

\begin{tabular}{|c|}
\hline LIPPINCOTT \\
WILLIAMS AND WILKINS
\end{tabular}

IOURNAL NAME

MEG

10/12/05

ARTICLE NO:

200066

\section{QUERIES AND / OR REMARKS}

\begin{tabular}{|c|c|c|}
\hline $\begin{array}{c}\text { Query } \\
\text { No }\end{array}$ & Details Required & Authors Response \\
\hline AQ1 & Please provide location of manufacturer Nutricia & \\
\hline $\mathrm{AQ} 2$ & Please provide manufacturer and precise location for Narco Bio System MMS 200 & \\
\hline $\mathrm{AQ3}$ & Please provide Conflicts of interest and Author's contributions statements & \\
\hline AQ4 & Ref $11-$ please provide full page range, or is 1 page only correct? & \\
\hline
\end{tabular}

